\title{
High-cadence measurements of electron pitch-angle distributions from Solar Orbiter SWA-EAS burst mode operations
}

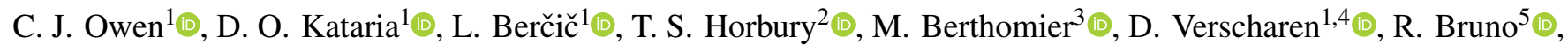

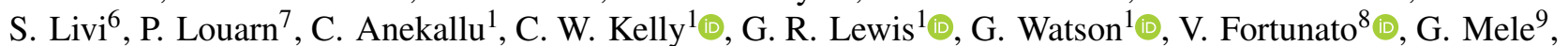
G. Nicolaou ${ }^{6,1}$, R. T. Wicks ${ }^{10}$, H. O’Brien $^{2}$, V. Evans ${ }^{2} \odot$, and V. Angelini ${ }^{2}$

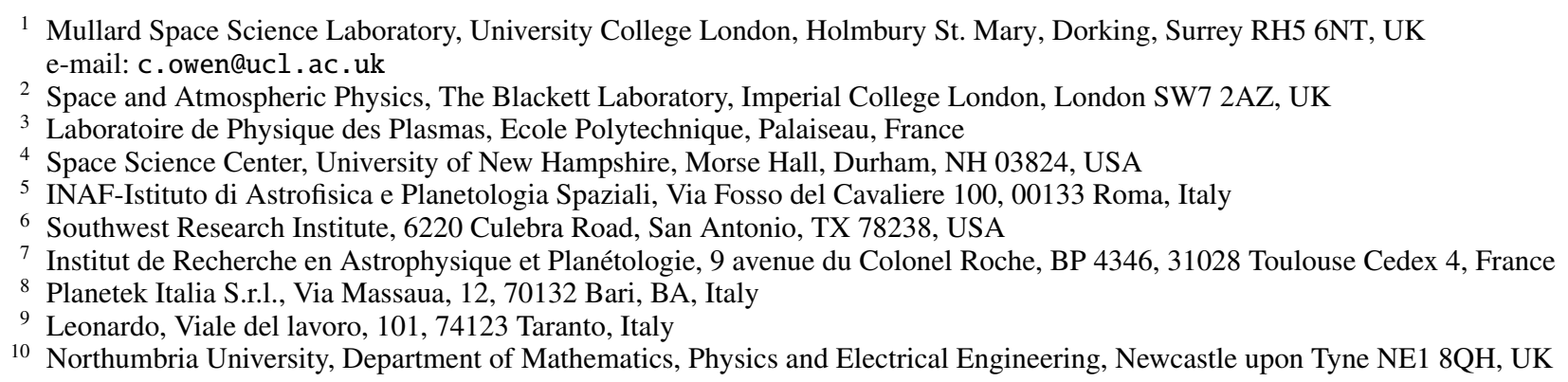

Received 31 March 2021 / Accepted 18 June 2021

\section{ABSTRACT}

\begin{abstract}
Small-scale kinetic processes associated with turbulence, plasma instabilities, magnetic reconnection, etc., play a major role in dissipating energy and governing the large-scale evolution of the solar wind. However, a general impediment to improving the understanding of the kinetic physics of the solar wind is in the limitations on measurement cadences of particle instruments, which are usually several orders of magnitude below the equivalent cadences of field instruments. Nevertheless, knowledge of the details of the particle velocity distribution functions (VDFs) at sub-second cadence is required to make progress in this area. This is particularly true for the electron VDFs, which play a significant role in the overall energetics of the solar wind through their transmission of heat flux from the Sun. In this paper, we detail and illustrate a novel measurement scheme deployed on Solar Orbiter's Solar Wind Analyser Electron Analyser System (SWA-EAS), which allows for 2D pitch angle distributions (PAD) to be returned over short periods (5-10 min) at a cadence of $0.125 \mathrm{~s}$. This is achieved through the use of a B-field vector shared by the magnetometer (MAG) instrument to steer the SWA-EAS system to record only that part of the full SWA-EAS field-of-view needed to construct the PAD. We provide an example of early observations using this scheme to illustrate that it is working well. Given that the electrons are usually gyrotropic, these measurements provide a new tool with which to derive details of the electron VDFs at high cadence for the study of the solar wind's kinetic processes.
\end{abstract}

Key words. magnetic fields - instabilities - plasmas - Sun: heliosphere - solar wind - methods: observational

\section{Introduction}

Many of the prime goals of the Solar Orbiter mission involve establishing links between the activity in the solar photosphere or corona and the phenomena subsequently observed in inner heliosphere space (Müller et al. 2020). To this end, the mission carries a comprehensive payload comprising both remote sensing (RS) instruments for diagnostics of the solar atmosphere and in situ (IS) instrumentation aimed at providing measurements of the solar wind in the vicinity of the spacecraft. Due to telemetry (TM) constraints, the RS instruments operate in observing windows that cover $\sim 30$ days in a $\sim 6$-month orbit. However, the IS instruments, comprising an energetic particle detector suite (EPD, Rodríguez-Pacheco et al. 2020), a magnetometer (MAG, Horbury et al. 2020), a radio and plasma waves instrument suite (RPW, Maksimovic et al. 2020, and a solar wind plasma analyser suite (SWA, Owen et al. 2020), each hold the capability of near full-time operation. Each of these can operate with temporal measurement cadences from seconds to minutes, which provide appropriate data to pursue Sun-heliosphere connection science topics. However, to fully exploit their high duty cycles for operation and to make best use of operations outside of the RS windows, the mission science activity plan (Zouganelis et al. 2020) also includes goals related to the intrinsic physics of the solar wind itself. These goals include advancing our understanding of physical processes such as turbulence, plasma instabilities, magnetic reconnection, collisionless shocks, and other kinetic plasma processes that, despite their operation on relatively small temporal and spatial scales, are involved in the complex dynamics driving both the large-scale thermodynamics and evolution of the solar wind as well as the localised transfer of energy between EM fields and charged particles (Verscharen et al. 2019).

Providing measurements on timescales appropriate for the study of kinetic plasma processes is generally not an issue for the EM fields and waves instruments, such as MAG and RPW, since they can relatively easily access the sub-second 
regime associated, for example, with the ion and electron dissipation range for solar wind turbulence (e.g., Goldstein et al. 2015, and references therein). These timescales are significantly more challenging to achieve for particle instrumentation, where obtaining a full 3D velocity distribution functions (VDF) measurement may be dependent on the spacecraft spin rate (typically of a few seconds) or on electrostatic deflection techniques combined with accumulation times, which allow for sufficient counts to be recorded in each measurement bin. This limitation has been overcome, for example, by the Magnetospheric Multiscale (MMS) mission (Burch et al. 2016), which deploys eight sets of particle detectors per spacecraft in order to address the physics of magnetic reconnection. However, this approach is not practical within the scope of most missions. Nevertheless, the determination of deviations in solar wind particle VDFs from the Maxwellian equilibrium state is critical to understanding the complex interplay between coronal heating, acceleration, global expansion, and local kinetic processes in driving the evolution of the solar wind plasma (e.g., Marsch 2006). Furthermore, a proper assessment of the kinetic microstate of the solar wind requires particle VDFs at the sub-second cadence accessible to the fields instruments. Fortunately, in the case of electrons, the gyrotropy of the VDF around the B-field direction is expected to be very good, such that the fast measurement of an electron 2D pitch angle distribution (PAD) can go a long way towards meeting these goals.

In this paper, we provide the details of a novel technique deployed on Solar Orbiter for making such high-cadence measurements of the electron PAD using the SWA Electron Analyser System (SWA-EAS). We first review the basic concept and operation of the sensor in Sect. 2. In Sect. 3, we describe the scheme and the on-board operations that allow the sensor to capture a complete 2D electron PAD on timescales of 0.125 s. In Sect. 4 , we provide an example of the operation of the scheme before discussing the caveats to its use. We provide a summary of our work in Sect. 5.

\section{SWA-EAS}

A description of the SWA-EAS structure and operation can be found within the SWA instrument paper (Owen et al. 2020). In this section, we summarise the relevant points pertaining to the fast capture of electron PADs.

Figure 1 shows a schematic of the SWA-EAS sensor, which is located on the end of a $4 \mathrm{~m}$ long boom that maintains its location within the spacecraft shadow. The rectilinear box structure contains most of the instrument electrical subsystems and provides the mounting for two separate cylindrical electrostatic analyser (EA) heads (e.g., Carlson et al. 1982), each with an aperture deflection system (ADS). The ADS is used to electrostatically steer electrons arriving from 'elevation' directions within $\pm 45^{\circ}$ of the centre plane (that lying orthogonal to the axis of cylindrical symmetry) of each sensor head into its aperture. Each sensor head simultaneously accepts electrons arriving from a full $360^{\circ}$ in azimuth, except for three minor blockages due to three pillars that mount the top of the sensor head to its lower half. Overall, each sensor head separates, within its own science frame, the direction of arrival of electrons into 32 bins in azimuth $\left(0^{\circ} \leq \phi<360^{\circ}\right)$ and 16 bins in elevation $\left(-45^{\circ} \leq \theta<45^{\circ}\right)$. We note that the 16 bins in elevation are not equally sized due to the asymmetry in the mounting environment between top and bottom of each head (see Owen et al. 2020 for further details). Since the two sensor heads are mounted orthogonally to each other (and at $45^{\circ}$ to the spacecraft-Sun line), the two

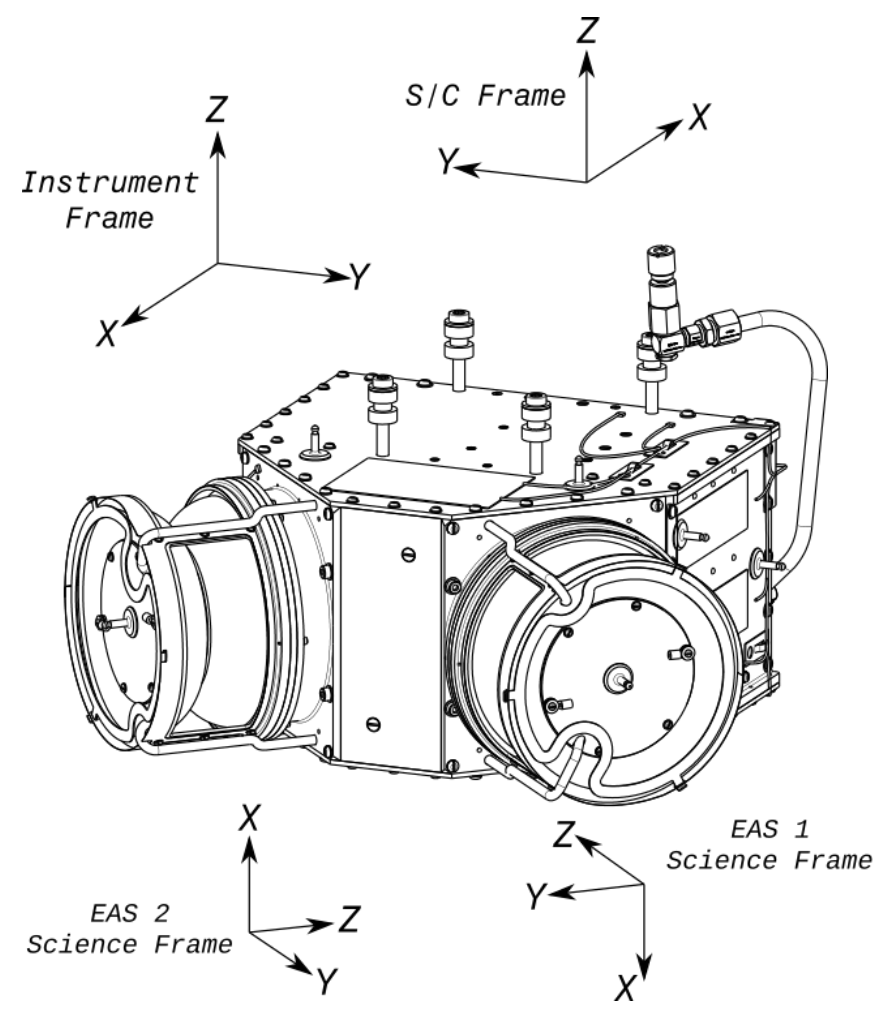

Fig. 1. Schematic of the SWA-EAS sensor. The rectilinear box houses sensor electronics and provides orthogonal mounting for two cylindrical sensor heads comprising an aperture deflection subsystem, a hemispherical electrostatic analyser subsystem, an annular microchannel plate detector/anode subsystem. Each head accumulates counts of electrons in 64 energy bins $(\sim 1 \mathrm{eV}$ to $\sim 5 \mathrm{keV})$ arriving from each of 16 elevation directions $\left( \pm 45^{\circ}\right)$ and 32 azimuth directions $\left(0^{\circ} \leq \phi<360^{\circ}\right)$. Accumulation time for a full 3D data product is $\sim 1 \mathrm{~s}$ (see Owen et al. 2020 for further details).

swaths of sky representing each sensor heads field of view (FoV) are combined to cover the full sky, with additional blockages only due to the spacecraft and its appendages. Within each sensor head, a high-heritage design for a hemispherical EA allows the separation of electrons entering the instrument aperture into 64 logarithmically-spaced energy bins ranging from $\sim 1 \mathrm{eV}$ to $\sim 5 \mathrm{keV}$.

Given that each sensor head accepts electrons arriving from all azimuths simultaneously, the mode of operation to build up a full 3D VDF is to use the ADS system to select a given elevation angle for electrons arriving into each head, then to sweep the EA across the full range of 64 energies, before moving on to the next elevation setting. The accumulation time for each elevationenergy bin measurement is $0.96 \mathrm{~ms}$, such that the full set of measurements is made in $16 \times 64 \times 0.96 \mathrm{~ms}=0.983 \mathrm{~s}$. Although the combined sensor pair are capable of producing the resulting full $3 \mathrm{D}$ measurement space at this $\sim 1$ s cadence, the volume of data involved is challenging for the SWA TM budget, even after compression is applied. For this reason, this full cadence data product can only be returned to the ground for very limited periods, at most 5 min per day on average. Rather, the SWA Data Processing Unit (SWA-DPU) uses the base 3D VDF to calculate onboard moments at a $4 \mathrm{~s}$ cadence, which is returned to the ground with the full VDFs returned at only 10 or $100 \mathrm{~s}$ cadence depending on TM availability. In any case, all of these available data cadences are $\geq 1 \mathrm{~s}$, which is already long compared to what is ideally required to address the solar wind kinetic physics 


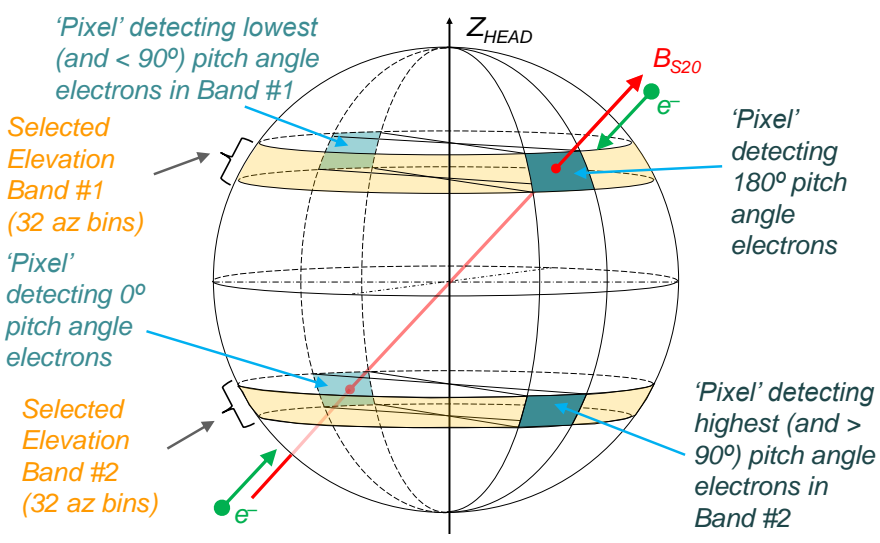

Fig. 2. Schematic of the rationale of the SWA-EAS BM PAD capture scheme. The sensor head with centre-plane closest to the B-field direction is steered to capture the two elevation bands containing the field direction and anti-field direction. In these two bands, all 32 azimuth bins record incoming electrons simultaneously. This then captures 64 samples in pitch angle space spread between $0^{\circ}$ and $180^{\circ}$.

topics outlined in the introduction. For this reason, we derived a 'burst mode' (BM) of operation, which improves this situation by capturing a $2 \mathrm{D}$ electron PAD at eight times the temporal resolution of the $3 \mathrm{D}$ product. In the next section, we describe the onboard algorithm used in this scheme.

\section{High-cadence PAD capture scheme}

In order to further the ability of the SWA-EAS sensor to contribute to the kinetic physics goals of Solar Orbiter, we devised a BM operational scheme, whereby we sample the minimum number of measurement elements required to provide a valid 2D PAD of the electrons at subsecond resolution. Fundamental to this operation is the availability to the SWA-DPU of the nearcontemporaneous B-field unit vector, $\hat{\boldsymbol{b}}$, from the MAG instrument (Horbury et al. 2020). This need was addressed by the MAG and SWA teams, together with ESA and Airbus UK, during the mission planning phase, so this data product is communicated between MAG and SWA onboard over an inter-experiment link, known as Service 20 (S20). This data product is communicated at $0.125 \mathrm{~s}$ cadence and with minimal latency.

On receipt of a magnetic unit vector, an algorithm within the SWA-DPU first calculates which of the two SWA-EAS sensor heads has the B-field direction lying closest to its aperture centre plane (equivalent to the largest angle between $\hat{b}$ and the sensor head science frame $Z$-axis shown in Fig. 1). For that sensor head, the algorithm calculates which of the 16 elevation bins contains $\hat{\boldsymbol{b}}$ and additionally which contains $-\hat{\boldsymbol{b}}$. A schematic illustrating this selection process is shown in Fig. 2. This figure represents the measurement sphere for a single energy, with the axis of cylindrical symmetry of the sensor head pointing up the page and marked as $Z_{\mathrm{HEAD}}$. We draw the projection of $\hat{\boldsymbol{b}}$ through this sphere as the red-arrowed line marked $B_{S 20}$. This line crosses the sphere at two points, marked by the red dots (we note that the fainter colouring and dashed lines are intended to represent elements of the figure which are below the surface of the sphere facing the viewer). These points are associated with specific azimuth and elevations in the sensor head reference frame, and therefore with two specific angular 'pixels' (represented by blue shading around the red dots) within the sensor head measurement space. We note that an electron travelling with pitch angle $180^{\circ}$ (i.e., antiparallel to the field direction, represented by the green arrow in the upper right quadrant) will be detected and associated with the pixel shaded blue in the top right. Conversely, an electron travelling with pitch angle $0^{\circ}$ (i.e., parallel to the field direction, represented by the green arrow in the lower left quadrant) will be detected and associated with the pixel shaded blue in the lower left of the figure. Thus, if the sensor is commanded to measure only these two specific angular bins, determined with reference to the $\mathrm{B}$-field direction, we can be sure that we have captured both the $0^{\circ}$ and $180^{\circ}$ pitch angle electrons. However, since all azimuths in the selected sensor head frame for a given elevation and energy are measured simultaneously, there is no time penalty to also capturing the other 31 azimuth pixels associated with the two selected elevations. These two selected elevation bands, each containing 32 azimuthal bins, are shaded in yellow in the figure.

We now consider the pitch angles associated with the two sets of 32 azimuth bins selected by this algorithm. For the 'selected elevation band \#1' shown in the upper part of Fig. 2, we note that as we move away from the azimuthal pixel containing the B-field direction and 'detecting' $180^{\circ}$ pitch angle electrons, the azimuth angle of the measurement corresponds to electrons of lower and lower pitch angles. The lowest pitch angle sampled in this band corresponds to the pixel azimuth directly opposite that containing the B-field (light blue shaded pixel in upper left quadrant of the figure). From the geometry, this always corresponds to pitch angles of $<90^{\circ}$, and if the selected band was at $0^{\circ}$ elevation (the case when $B_{S 20}$ is perpendicular to $Z_{\mathrm{HEAD}}$ ), then this pixel would measure electrons with $0^{\circ}$ pitch angle. Conversely, for 'Selected Elevation Band \#2' in the lower part of Fig. 2, azimuthal pixels located increasingly further away from that collecting $0^{\circ}$ pitch angle electrons correspond to measurement of electrons at increasingly higher pitch angles. Similarly to arguments above, the highest pitch angle detected in this band will be $>90^{\circ}$. Thus, selecting just the two elevation bands illustrated in Fig. 2 will provide two sets of 32 pitch angles, $\alpha_{1}, \alpha_{2}$ with ranges $\alpha_{1}=\left[<90^{\circ}: 180^{\circ}\right]$ and $\alpha_{2}=\left[0^{\circ}:>90^{\circ}\right]$. Thus, between the two selected bands, we have 64 measurements of the electrons with pitch angle range from $\left[0^{\circ}: 180^{\circ}\right]$.

We further illustrate the operation of this algorithm by presenting a specific example in a heliospheric coordinate system. Figure 3 shows a 'map' of the full sky covering $\pm 180^{\circ}$ in azimuth and $\pm 90^{\circ}$ in elevation. In this format, we may locate the Sun at $[0,0]$ (corresponding to $[1,0,0]$ in the spacecraft frame shown in Fig. 1) and $+90^{\circ}$ elevation points northward out of the plane of the spacecraft orbit $([0,0,1]$ in the spacecraft frame). As a definitive example, we represent the case in which the $\mathrm{B}$-field direction is located at azimuth $32^{\circ}$ and elevation $15^{\circ}$, as marked by the red diamond symbol. The direction opposite to the field direction is then marked by the red triangle at $\left[-148^{\circ},-15^{\circ}\right]$. This field direction falls within the FoV of the EAS1 sensor head but is outside of the region sampled by EAS2. We therefore show, as the blue grid, the pixelated FoV of the EAS1 sensor head transformed onto this sky map. Selection of an elevation band containing the FoV means we capture all data from the region shaded orange and passing across the red diamond symbol. The selection of the elevation band containing the anti-field aligned direction captures the data from the orange band passing through the triangle symbol (and wrapping across the left and right edges of the plot). Superimposed over the plot are a set of red contours, which represent the loci for particles arriving with $30^{\circ}, 60^{\circ}, 90^{\circ}$, $120^{\circ}$, and $150^{\circ}$ pitch angles for this orientation of the B-field. We note that the first selected band crosses pitch angle contours from $180^{\circ}$ (red diamond) down to $<30^{\circ}$ (near $\left[-120^{\circ},-15^{\circ}\right]$ ), 


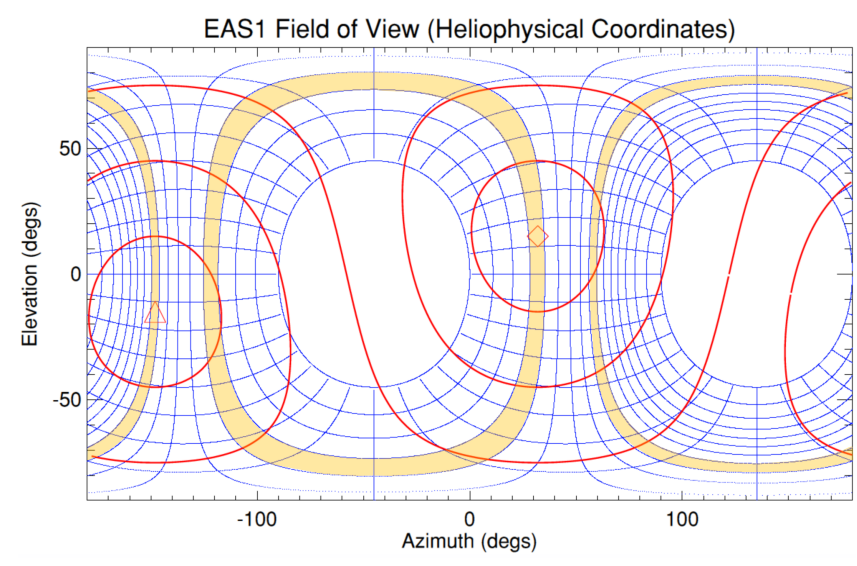

Fig. 3. Full sky map showing an example of the SWA-EAS BM capture scheme. The B-field direction is chosen here to be at $\left[32^{\circ}, 15^{\circ}\right]$ (red diamond) so the anti-field direction is at $\left[-148^{\circ},-15^{\circ}\right]$ (red triangle). The blue grid shows the projection of the EAS1 angle-angle bins onto the plane of the sky, while the red contours show lines of constant pitch angle for this B-field direction, for pitch angles of $30^{\circ}, 60^{\circ}, 90^{\circ}, 120^{\circ}$, and $150^{\circ}$. The two selected elevation bands are shown shaded in orange. Combined these cross the full range of pitch angle space.

while the second crosses contours from $0^{\circ}$ (red triangle) up to $>150^{\circ}$ (near $\left[60^{\circ}, 15^{\circ}\right]$ ). This again illustrates that, in principle, a full range of the electron PAD is sampled.

A key point here then is that since we are able to obtain a full PAD by steering just one of the two sensor heads to sample only the two elevations containing the B-field and anti-field directions. Since the full 16 elevations comprising the full 3D VDF is not recorded in this mode, we are able to reduce the sample time by a factor of 8 and the data volume per sample by a factor of 16. In practice, this means that we can capture and return this data product at a time cadence of $0.125 \mathrm{~s}$. However, the data volume per second, which contains 8 times as many samples, is only half that of the 3D sample. Hence, the deployment of this mode in practice must also be limited to only a few tens of minutes per day. In the next section, we present an example of the data returned from SWA-EAS deployed in this mode.

\section{Example BM Event}

Figure 4 shows an example of data returned from an SWA-EAS BM on 24 June 2020, lasting for 5 min from 05:33:34 UT. The top panel shows the components of the B-field unit vector, as supplied to SWA-EAS by MAG via the S20 link, in the spacecraft frame (cf. Fig. 1). In this panel, the $x$-component is shown as the red trace, the $y$-component in green and the $z$-component in magenta. The next two panels show the elevation and azimuth angle of the B-field vector in the EAS1 (red) and EAS2 (yellow) science frames. We note, however, that the yellow trace is absent from the second panel for much of the period shown since the elevation angle in this frame moves below $-45^{\circ}$ and thus outside the EAS 2 FoV covering $\pm 45^{\circ}$. The second panel also shows a series of magenta boxes which show the extent of the elevation bin selected by the B-field tracking algorithm as that containing the field, along with a set of light blue boxes showing the extent of the elevation bin selected to contain the anti-field direction. It can be seen here that the EAS1 elevation trace (red) remains almost entirely within the selected elevation boxes, despite variations of elevation covering several tens of degrees.

Panel 4 shows the sensor head used for the selection of each data point in the time series. For the most of the event, the field

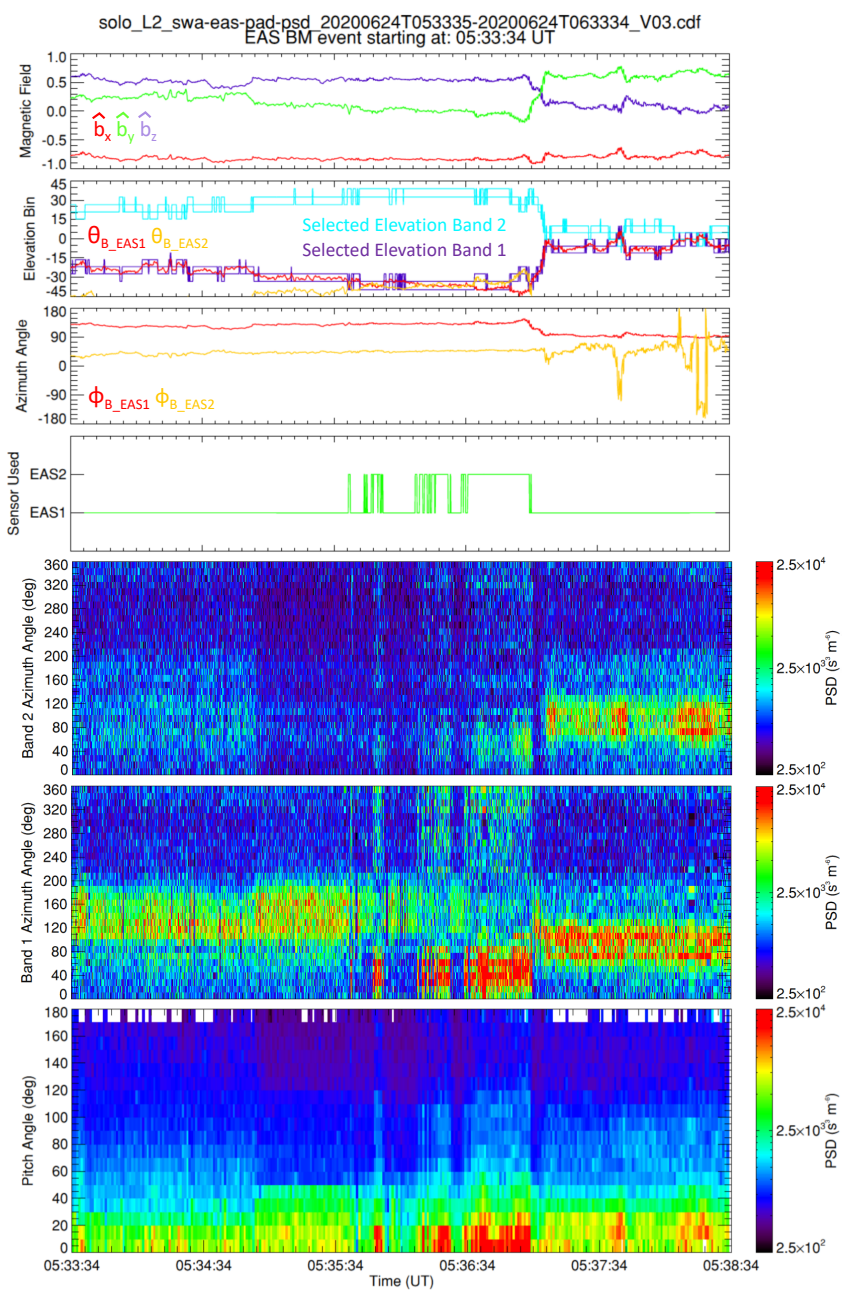

Fig. 4. Example of an SWA-EAS BM event, showing $5 \mathrm{~min}$ of data recorded on 26th June 2020. Top panel: B-field unit vector received over S20 from MAG. Panel 2: illustrates the selection of the EAS elevation bins for each sample, based on the MAG data. Panel 3: azimuth of the field in the 2 SWA-EAS science frames, while panel 4 indicates the sensor head selected to supply data. Panels 5 and 6: contain the data recorded in the elevation bins containing the field and anti-field aligned directions for each measurement sample. These data, for electrons of energy $>70 \mathrm{eV}$, are recorded as a function of azimuth at $0.125 \mathrm{~s}$ cadence. Bottom panel: data rebinned into pitch angle space, showing that a full PAD was obtained for most of the duration of the event. See text for details.

direction is closest to the centre of the EAS1 FoV, so that head is selected to provide data. However, during the period 05:34:40 05:36:20 UT, the field direction moves also into the EAS2 FoV, such that the orange trace is visible, and for some short periods is closer to zero elevation in the EAS2 frame than in the EAS1 frame. At those times, the data selection switches from EAS1 to EAS2, as indicated in the fourth panel. We note that after 05:37:00 UT, the B-field orientation switches such that it lies close to the EAS1 FoV centre plane (near $0^{\circ}$ elevation in the EAS1 science frame) and is almost aligned with the axis of symmetry for EAS2 (not shown) where the field elevation angle is $\sim 90^{\circ}$. Thus, for this period, small shifts in the direction of the field result in the large azimuthal shifts seen in the orange trace in panel 3. Overall however, these upper four panels of Fig. 4 show that the algorithm for selecting the appropriate sensor head and the appropriate pair of elevations works very well for these inputs.

Panels 5 and 6 show the time series of data collected in the two selected elevation bins as a function of azimuth angle and 
time through the BM event. Here, we opted to present the averaged phase space density for all energy bins $>70 \mathrm{eV}$, shown at full $0.125 \mathrm{~s}$ cadence. We note that data exists for electrons of lower energies, but at the time of writing, there is a need to remove the contamination from spacecraft-generated electrons. Moreover, the energy range shown here is representative of the solar wind electron strahl, which is expected to show the greatest degree of ordering by the B-field (Feldman et al. 1975).

Panels 5 and 6 show that the Band 1 fluxes are relatively low for the first half of the event, while in Band 2 there is a swathe of generally higher fluxes initially centred around $>150^{\circ}$ azimuth and strengthening and shifting to lower azimuths later in the period shown. However, a higher order data product is obtained by associating each of the $2 \times 32$ azimuths shown in panels 5 and 6 with the corresponding pitch angle and combining measurements in pitch angle space. The result of this exercise for this case is shown in the bottom panel of Fig. 4, in which we have resampled the data into one of 18 10-degree wide pitch angle bins and also summed to a $1 \mathrm{~s}$ time cadence for clarity of presentation. This data then shows the complex variation in the strahl fluxes immediately before and after the significant B-field rotation at 05:37:00 UT. Although the strahl fluxes in the field aligned direction remain dominant throughout the event, despite the field rotation, the SWA-EAS data captured clearly shows the short timescale variations in both the intensity of the beam and its pitch-angle width, including significant variations observed across the current sheet itself. We believe this demonstrates a new observational capability through which short timescale interactions between the fields and electrons can be studied to bring new light on kinetic scale processes. Indeed, we note that this capability has been used by Berčič et al. (2021) to examine the evolution of the strahl electron PAD observed later the same day during the presence of whistler waves detected by the RPW experiment (Maksimovic et al. 2020).

\section{Discussion and conclusion}

In this paper, we have described and illustrated the operation of a novel on board algorithm, which facilitates the rapid acquisition of electron PADs from the Solar Orbiter SWA-EAS. Although the scheme works well in general, there are a number of caveats to be kept in mind when analysing the resultant data.

Firstly, the sampling of a PAD in principle loses information related to the gyrotropy of the full VDF, which may be a drawback in some kinetic contexts (e.g., near a reconnection region). However, our scheme does sample the underlying VDF at multiple gyrophase angles, particularly for more perpendicular pitch angles. Thus, limited gyrophase information is still recoverable.

Secondly, although the tracking of the B-field vector received over the S20 link is excellent, the quality and relevance of the resulting PAD can only be as good as the quality and relevance of the input data. Although this is generally excellent, there are known issues that may affect the quality of the B-field information, such as electromagnetic contamination from spacecraft subsystems and other instruments, as well as drifts of the MAG offsets. If any such issues cause the steering algorithm to depart from the actual B-field location by the width of one SWA-EAS elevation bin (or more), then the PAD completeness will be compromised. In addition, there is, by necessity, a latency in the transmission of data from the MAG instrument, which comes via the spacecraft central computer and the SWA-DPU to the EAS sensor. This latency is likely to be somewhat variable depending on the traffic on the S20 link. This will have impact on the resulting science data if the field vector moves significantly (again by more than the width of one SWA-EAS elevation bin) during the period of latency. If this is the case, then the SWA-DPU may steer the EAS using a field vector that is no longer relevant.

If either of the above issues become significant, there is a resulting impact on the completeness of the science data product, although not necessarily the overall quality of the data obtained. If the steering is in error by more than the width of an elevation bin, then the foremost consequence is the failure to capture the $0^{\circ}$ and $180^{\circ}$ pitch angle particles. Given the average half-width of an SWA-EAS elevation bin is $\sim 2.8^{\circ}$, we would typically lose these field-aligned measurements if the elevation of the onboard B-field vector were in error by that amount. However, the data that is returned can be rebinned on the ground according to validated ground MAG data, so that accurate, albeit incomplete, PAD scans can still be obtained. It is anticipated that the groundvalidated, rebinned PAD's, at 0.125 s resolution, will be a regular SWA Level 3 data product available from the Solar Orbiter Archive $^{1}$.

In summary, we have deployed an important new tool for the study of solar wind electrons and their interactions with electromagnetic fields and waves. Electrons are highly mobile and carry a substantial heat flux into the solar system. Thus, attaining an understanding of both how that heat flux is carried into the heliosphere by the creation of suprathermal features within the distribution and how it is dissipated via kinetic processes operating on small timescales is key to understanding the overall energetics of the plasma. While it is unlikely that particle instruments will soon achieve the kind of time resolution for measurements that are currently available through EM fields instruments, the algorithm described and illustrated here will shift the boundaries of what is possible with the application of particle data.

Acknowledgements. Solar Orbiter is a space mission of international collaboration between ESA and NASA, operated by ESA. Solar Orbiter Solar Wind Analyser (SWA) data are derived from scientific sensors which have been designed and created, and are operated under funding provided in numerous contracts from the UK Space Agency (UKSA), the UK Science and Technology Facilities Council (STFC), the Agenzia Spaziale Italiana (ASI), the Centre National d'Etudes Spatiales (CNES, France), the Centre National de la Recherche Scientifique (CNRS, France), the Czech contribution to the ESA PRODEX programme and NASA. Solar Orbiter SWA work at UCL/MSSL is currently funded under STFC grants ST/T001356/1 and ST/S000240/1. The Solar Orbiter MAG was funded by the UK Space Agency (grant ST/T001062/1). D.V. is supported by STFC Ernest Rutherford Fellowship ST/P003826/1. T.H. is supported by STFC grant ST/S000364/1.

\section{References}

Berčič, L., Verscharen, D., \& Owen, C.J. 2021, A\&A, 656, A31 (SO Cruise Phase SI)

Burch, J. L., Moore, T. E., Torbert, R. B., \& Giles, B. L. 2016, Space Sci. Rev., 199,5

Carlson, C., Curtis, D., Paschmann, G., \& Michel, W. 1982, Adv. Space Res., 2, 67

Feldman, W., Asbridge, J., Bame, S., Montgomery, M., \& Gary, S. 1975, J. Geophys. Res., 80, 4181

Goldstein, M. L., Wicks, R. T., Perri, S., \& Sahraoui, F. 2015, Phil. Trans. R. Soc. A Math. Phys. Eng. Sci., 373, 20140147

Horbury, T.S., OBrien, H., \& Carrasco Blazquez, I. 2020, A\&A, 642, A9

Maksimovic, M., Bale, S. D., Chust, T., et al. 2020, A\&A, 642, A12

Marsch, E. 2006, Liv. Rev. Sol. Phys., 3, 1

Müller, D., Cyr, O.C.St, \& Zouganelis, I. 2020, A\&A, 642, A1

Owen, C. J., Bruno, R., Livi, S., et al. 2020, A\&A, 642, A16

Rodríguez-Pacheco, J., Wimmer-Schweingruber, R. F., Mason, G. M., et al. 2020, A\&A, 642, A7

Verscharen, D., Klein, K. G., \& Maruca, B. A. 2019, Liv. Rev. Sol. Phys., 16, 5 Zouganelis, I., De Groof, A., Walsh, A. P., et al. 2020, A\&A, 642, A3

http://soar.esac.esa.int/soar/ 